\title{
AC002454.1 and CDK6 synergistically promote endometrial cell migration and invasion in endometriosis
}

\author{
Jing Liu, Yang Wang, Peng Chen, Yue Ma, Shuo Wang, Ye Tian, Anna Wang and Danbo Wang \\ Department of Gynecology, Cancer Hospital of China Medical University, Liaoning Cancer Hospital and Institute, \\ Shenyang, Liaoning, People's Republic of China
}

Correspondence should be addressed to D Wang; Email: wangdanbo@cancerhosp-In-cmu.com

\begin{abstract}
Previous IncRNA microarray screening found that the AC002454.1 gene was highly expressed in endometriosis (EMS), and these expression levels were highly correlated with cyclin-dependent kinase-6 (CDK6). This study investigated the expression level and correlation between AC002454.1 and CDK6 in endometrium tissues and the influence of these changes in expression upon the biological behavior of eutopic endometrial cells. We confirmed AC002454.1 and CDK6 mRNA and protein were highly expressed in ectopic and eutopic endometrial tissue from patients with EMS and were clearly correlated. In vitro, both AC002454.1 and CDK6 positively regulated the proliferation, migration and invasion ability of eutopic endometrial cells and could promote the transformation of cells from G0/G1 phase to S phase. AC002454.1 and CDK6 may have synergistic effects, thereby affecting the biological behavior of endometrial cells, and thus promote the progression of EMS.

Reproduction (2019) 157 535-543
\end{abstract}

\section{Introduction}

Endometriosis (EMS) is an estrogen-dependent disease which is refractory and associated with cell dysfunction. The main clinical symptoms of EMS are chronic pelvic pain, infertility and dysmenorrhea (Lessey \& Kim 2017), which are associated with extensive maturation, invasion and a growth ability similar to a malignant tumor (Giudice \& Kao 2004). The retrograde menstruation phenomenon represents a classic theory relating to the pathogenesis of EMS (Burney \& Giudice 2012). Although 90\% of women experience menstrual blood flow during menstruation, only $10 \%$ of women suffer from this disease (Mckinnon et al. 2018), indicating that the inherent biological characteristics of the eutopic endometrium must play a key role (Xu et al. 2010). Studies have shown that patients with EMS in the lining of the endometrium, especially during the secretory phase, appear different in terms of endometrial cell structure, migration, invasion, proliferation, angiogenesis, apoptotic ability and the expression of certain genes (Sundqvist et al. 2012). These studies have provided further evidence for 'eutopic endometrium determinism' (Zhang et al. 2015).

Previous preliminary work by our group used IncRNA chip screening and bioinformatic analysis to investigate the endometrium in both EMS and normal subjects. This work found that the AC002454.1 gene (Transcript ID: ENST00000452050) is one of the most highly expressed genes; real-time polymerase chain reaction (RT-PCR) confirmed that the relative expression of AC002454.1
mRNA in eutopic endometrium tissue (EU) was significantly increased (Wang et al. 2015).

LncRNAs form a group of RNAs which are more than 200 nucleotides in length, lack a complete open reading frame, have no protein-coding function and are ubiquitous in eukaryotic cells (Khorkova et al. 2015). LncRNAs are known to play an important role in malignant tumors such as gastric cancer (Yan et al. 2016), prostate cancer (Ling et al. 2017), lung cancer (Loewen et al. 2014) and kidney cancer (MartensUzunova et al. 2014). AC002454.1 was previously identified as a natural antisense IncRNA (Wang et al. 2015). In our previous chip analysis, we found that AC002454.1 exhibits a significant association with its adjacent gene, cyclin-dependent kinase-6 (CDK6) (Pearson's correlation coefficient: 0.744, $P<0.001$ ). AC002454.1 is located at position 92465802-92546437 of chromosome 7, while our previous work showed that CDK6 was the mRNA with the highest fold difference in the cell cycle pathway and was ranked first in our pathway analysis (Wang et al. 2015). CDK6 is located at position 92234234-92465941 of chromosome 7, both AC002454.1 and CDK6 have overlapping regions of approximately 139 nucleotides in length. The positional relationship between AC002454.1 and CDK6 is head-to-head; in other words, the $5^{\prime}$ end sequence of the antisense strand is complementary to the $5^{\prime}$ end sequence of the sense strand.

CDK6 is a carcinogenic kinase that regulates the cell cycle in the G1 phase (Tigan et al. 2016) and initiates 
transcription of the genes necessary for cell cycle and cell proliferation (Paternot et al. 2014). Gene ontology analysis suggested that differential mRNA transcripts are primarily associated with the cell cycle (Wang et al. 2015). In addition to promoting cell proliferation, CDK6 also enhances vascular endothelial growth factor A (VEGF-A) expression, thereby inducing angiogenesis and endothelial cell growth, and thus, enhances vascular permeability; this action is independent of its kinase activity (Otto \& Sicinski 2013).

Previous studies have also confirmed that CDK6 and AC002454.1 are highly expressed in the endometrium of patients with EMS and show strong positive correlations (Wang et al. 2015). At the same time, bioinformatic prediction methods suggest that there may be direct interactions between AC002454.1 and CDK6 based on RNA-Protein Interaction Prediction (RPISeq). Therefore, it is reasonable to speculate that these two genes may complement each other to form an IncRNA-mRNA duplex and participate in maintaining the stability of CDK6-mRNA and thus increase protein expression levels. This causes cell cycle disorder in endometrial cells, causing changes in their proliferative capacity and angiogenic ability, thus participating in the disease progression of EMS.

The regulation of CDK6 and AC002454.1 may represent an important molecular mechanism in the formation of a hyperproliferative state during the secretory phase of the endometrium in patients with EMS. However, in the context of EMS, there is still a lack of research relating to IncRNA and CDK6 in terms of cell cycle disorder. In the present study, we aimed to investigate the expression, correlation and regulatory relationships of AC002454.1 and CDK6 in ectopic and eutopic endometrium from patients with EMS. We also investigated the effect of AC002454.1 and CDK6 in the biological behavior of eutopic endometrial cells from patients with endometriosis.

\section{Materials and methods}

\section{Tissue sample collection}

After obtaining consent from the Liaoning Cancer Hospital Medical Ethics Committee (Ethical approval number: 20170222) and written informed consent from patients, we selected endometrial and ectopic lesions from the secretory phase of patients with EMS according to preoperative ultrasonography and menstrual cycle data as the experimental group. Subjects with a normal endometrium and without estrogen-dependent diseases served as a control group. Thirty specimens from the experimental and control groups were used for tissue experiments, while ten specimens were used for cell experiments. The clinical characteristics of patients from the experimental group and the control group were not significantly different $(P>0.05)$, including age (mean age: $37.53 \pm 6.54$ years in the EMS group and $40.30 \pm 5.10$ years in the control group) and BMI (mean: $21.42 \pm 2.24$ in the EMS group and $21.83 \pm 3.39$ in the control group). All patients had regular menstruation and no other malignant, estrogendependent, immune, surgical or inflammatory diseases. Furthermore, none of the subjects had received $\mathrm{GnRH}$ analogs, hormones or anti-inflammatory drugs for the 6 months prior to surgery. Each experiment was repeated at least three times.

\section{RT-PCR}

Total RNA extraction and transcription was performed in strict accordance with guidelines provided with the RNA Extraction and Transcript Kit (TaKaRa). The primer sequences are presented in Table 1. RT-PCR reactions (SYBRßPrmix Ex Taq ${ }^{\text {TM }}$ kit, TaKaRa) were performed on a LightCycler 480 (Roche) by denaturation at $95^{\circ} \mathrm{C}$ for $5 \mathrm{~min}$ ( 1 cycle) and amplification at $95^{\circ} \mathrm{C}$ for $10 \mathrm{~s}$ and then $60^{\circ} \mathrm{C}$ for $30 \mathrm{~s}$ ( 45 cycles). Quantitative mRNA expression was calculated using the $2^{-\Delta \Delta \mathrm{Ct}}$ method.

\section{Immunohistochemistry}

Immunohistochemistry (IHC) was performed using the SP kit (ZSGB-BIO, Beijing, China), in accordance with the manufacturer's instructions. Tissue sections were incubated with a CDK6 antibody (Santa Cruz; 1:200) and antibody binding was developed using a DAB chromogenic kit (Beyotime, Nanjing, China). For analysis, the sections were randomly numbered, double-blinded and analyzed by light microscopy (E100; Nikon). Analysis was performed independently by two pathologists; their consensus was considered as the final score. The extent of positive staining was scored by calculating the proportion (\%) of positively stained cells: $<5 \%$, 0 points; $5-10 \%, 1$ point; $10-50 \%, 2$ points and $>50 \%, 3$ points. The intensity of staining was scored as follows: no staining, 0 points; light yellow, 1 point; moderate yellow, 2 points and strong yellow, 3 points. The final IHC score was determined by multiplying the extent of the staining by the intensity; scores of 0-3 and 4-9 were considered negative and positive expression, respectively (Lv et al. 2018).

\section{Western blotting}

Total protein extraction was performed using protein lysate buffer in accordance with the manufacturer's instructions

Table 1 Primer sequences for real-time PCR.

\begin{tabular}{llll}
\hline \multirow{2}{*}{ Gene } & \multicolumn{2}{c}{ Primers sequences $\left(5^{\prime} \rightarrow 3^{\prime}\right)$} & Length $(\mathrm{bp})$ \\
\cline { 2 - 3 } AC002454.1 & Forward & Reverse & 123 \\
CDK6 & ACTGCAGCAAGGAAAAGGAAA & CAGACAAGAGGCATCATCAGC & 139 \\
GAPDH & GCCTCTTTTTCGTGGAAGTT & AATTGGTTGGGCAGATTTTG & 138 \\
\hline
\end{tabular}


(Beyotime). Protein concentration was determined using a BCA Protein Assay Kit (Beyotime). Protein samples $(80 \mu \mathrm{g})$ were resolved by $8 \%$ SDS-PAGE and transferred to PVDF membranes (Millipore) by wet transfer. Then 5\% skimmed milk powder was used to block non-specific binding sites at room temperature for $2 \mathrm{~h}$. Membranes were then incubated with CDK6 antibody (1:200), using a GAPDH antibody (1:10000, Proteintech, Chicago, IL, USA) as an internal reference, overnight at $4^{\circ} \mathrm{C}$. The following morning, sections were incubated with a corresponding secondary antibody (1:2000, Proteintech) for $120 \mathrm{~min}$ at room temperature. Finally, the Enhanced ECL kit (Beyotime) was used to develop positive signals and the integrated density was analyzed using ImageJ software.

\section{Primary culture of endometrial stromal cells}

Using an established culture method for mature endometrial stromal cells (Xu et al. 2010), fresh endometrial tissue was cut into $0.5-\mathrm{Imm}^{3}$ pieces and digested with $0.1 \%$ Type I collagenase digest (Sigma). Tissue residue was removed by filtration through a 100-mesh screen and then a 400-mesh screen. Finally, cells were cultured in configuration medium (CM) including DMEM/F12 (Hyclone, Logan, UT, USA) medium, 10\% fetal bovine serum (FBS, Bioind, Israel) and 1\% double antibiotic (Gibco) in an incubator containing 5\% $\mathrm{CO}_{2}$ at $37^{\circ} \mathrm{C}$. The primary cells were passaged three times in order to optimize the quality of the results.

\section{Lentiviral transfection}

Eutopic endometrial cells from patients with EMS (ESC) were divided into three groups: transfected with AC002454.1 and CDK6 silencing lentiviral particles (Genechem, China) (AC002454.1-RNAi, CDK6-RNAi), transfected with negative lentiviral particles (AC002454.1-NC, CDK6-NC) and a blank control group (ESC). Normal endometrial cells from a control group (NESC) were divided into three groups: transfected with AC002454.1 and CDK6 overexpressing virus (AC002454.1-CDNA, CDK6-CDNA), transfected with negative virus (AC002454.1-NC, CDK6-NC) and a blank control group (NESC).

Transfection was performed according to the instructions provided by Genechem. When the degree of fusion between endometrial stromal cells in each six-well plate reached $50 \%$, replaced the $\mathrm{CM}$ and added polybrene at a final concentration of $5 \mu \mathrm{g} / \mathrm{mL}$, and the multiplicity of infection value was 30 when infection was performed.

\section{Cell proliferation assays}

Cells from each group were trypsinized and seeded into a 96-well plate (5000 cells/well); cell-free medium was used as a blank control group. After transfection for 24, 48, 72 and $96 \mathrm{~h}$, we added $10 \mu \mathrm{L}$ of Cell Counting Kit8 (CCK8, Dojindo, Japan) reagent to each well and incubated at $37^{\circ} \mathrm{C}$ for $150 \mathrm{~min}$. The absorbance was then measured at a wavelength of $450 \mathrm{~nm}$ using an ELISA plate reader (Bio-Rad). The cell proliferation rate was calculated as the ratio of the optical density values.

\section{Wound-healing assays}

Cells from each group were transfected into a six-well plate and cultured until they reached $90 \%$ confluence. A wound was created with a pipette tip and cultured with serum-free DMEM/F12 medium. According images captured using an inverted microscope (Nikon) at 0, 24 and $48 \mathrm{~h}$, we determined the migration ability of the cells by counting the number of cells migrating into the scratches.

\section{Transwell assays}

Matrigel (BD) was diluted 1:6 with serum-free DMEM/ F12 medium, coated on the bottom of a Transwell chamber (Corning) and coagulated at $37^{\circ} \mathrm{C}, 5 \% \mathrm{CO}_{2}$ for $4 \mathrm{~h}$. After transfection for $48 \mathrm{~h}$, cells were diluted to $4 \times 10^{5} \mathrm{cells} / \mathrm{mL}$ in serum-free DMEM/F12 medium and inoculated $200 \mu \mathrm{L}$ into the upper chamber. Then, $600 \mu \mathrm{L}$ of $20 \%$ FBS medium was added to the lower chamber as a chemoattractant. The culture chamber was then removed and washed twice with phosphate buffer saline after $24 \mathrm{~h}$. We then wiped off cells from the upper layer of the membrane and fixed these in $4 \%$ paraformaldehyde for $20 \mathrm{~min}$ and stained with crystal violet at room temperature for $30 \mathrm{~min}$. Excess dye solution was then washed off and the cells were photographed using light microscopy.

\section{Cell cycle assay}

After transfection for $48 \mathrm{~h}$, cells from each group were collected and adjusted to a cell concentration of $1 \times 10^{6} / \mathrm{mL}$. Experiments involved $1 \mathrm{~mL}$ of single cell suspension and was carried out in strict accordance with the manufacturer's instructions (Keygen Biotech, Nanjing, China). Cell content was then evaluated by flow cytometry (BD Accuri C6), and the relative proportion of cells in G0/G1, S and G2/M phases was analyzed by ModFit LT software.

\section{Statistical analysis}

Statistical data analysis was performed using SPSS 20.0 statistical software (SPSS Inc.). Two independent sample $t$-tests were used to compare the differences between the two groups. One-way ANOVA was used to compare means between multiple groups. All data were expressed as mean \pm standard deviation. Correlation analysis was performed using Pearson's correlation analysis.

\section{Results}

\section{Expression of AC002454.1 and CDK6 mRNA}

Using GAPDH as an internal reference, RT-PCR indicated that the relative expression of AC002454.1 and CDK6 mRNA in EU were significantly higher than that of normal endometrium tissues (NE) $(P<0.05$; Fig. $1 \mathrm{~A}$ and $\mathrm{B}$ ) and were positively correlated (Pearson correlation coefficient: $0.691, P<0.001)$. There was no significant difference between ectopic endometrium tissue (EC) and EU $(P>0.05)$. 


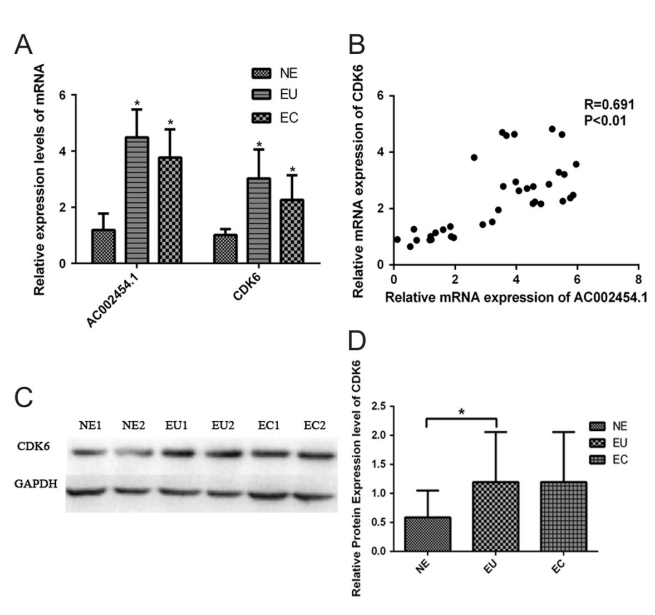

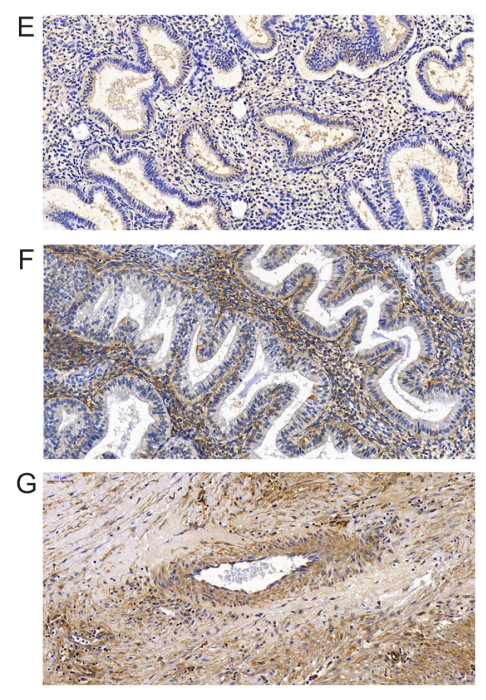

Figure 1 Expression of AC002454.1 and CDK6 in tissues. (A and $B$ ) The expression and correlation of AC002454.1 and CDK6, as detected by RT-PCR. $* P<0.05$ vs normal endometrium (NE). Pearson correlation coefficient: 0.691, $P<0.001$. (C) Western blotting of CDK6 proteins. (D) Relative protein expression levels of CDK6. ${ }^{*} P<0.05$ vs NE. (E, $F$ and $G$ ) Representative images of CDK6 staining, as detected by IHC. (E) NE; (F) eutopic endometrium tissue (EU); (G) ectopic endometrium tissue (EC). (magnification, $\times 200$ ).

\section{Expression of CDK6 protein}

Western blotting (Fig. 1C and D) showed that the relative expression of CDK6 protein in EU was significantly higher than that in NE $(P<0.05)$. There was no statistical difference between $\mathrm{EC}$ and $\mathrm{EU}(P>0.05)$.

IHC suggested that CDK6 protein was mainly expressed in the cytoplasm and nucleus of stromal cells in NE, EU and EC (Fig. 1E, F and G). The positive rate of CDK6 protein expression was $25.0 \%$ in NE, $66.7 \%$ in EC and $73.3 \%$ in EU. The positive expression rate of CDK6 protein in EU was significantly higher than that of NE $(P<0.05)$, while there was no significant difference between EC and EU $(P>0.05$; Table 2$)$.

\section{Overexpression of AC002454.1 and CDK6 promote expression of AC002454.1 and CDK6 mRNA}

RT-PCR results indicated that the relative expression levels of AC002454.1 and CDK6 mRNA in AC002454.1RNAi and CDK6-RNAi were significantly lower than those in AC002454.1-NC, CDK6-NC and ESC $(P<0.05$; Fig. 2A and B). The relative expression levels of AC002454.1, CDK6 mRNA in AC00254.1-CDNA and CDK6-CDNA were significantly higher than those in AC002454.1-NC, CDK6-NC and NESC $(P<0.05$; Fig. $2 \mathrm{C}$ and $\mathrm{D})$. There was no significant change between the negative lentiviral particles group and the blank control group $(P>0.05)$.

\section{Overexpression of AC002454.1 and CDK6 promotes the expression of CDK6 protein}

Western blotting results suggested that the relative expression level of CDK6 protein in AC002454.1-RNAi and CDK6-RNAi were significantly lower than those in AC002454.1-NC, CDK6-NC and ESC $(P<0.01$; Fig. 2E and $G$ ). The relative expression levels of CDK6 protein in AC00254.1-cDNA and CDK6-cDNA were significantly higher than those in AC002454.1-NC, CDK6-NC and NESC $(P<0.05$; Fig. $2 \mathrm{~F}$ and $\mathrm{H})$. There was no significant change between the negative lentiviral particles group and the blank control group $(P>0.05)$.

\section{Overexpression of AC002454.1 and CDK6 promote proliferation of endometrial stromal cells}

Under basal conditions, ESC exhibited more vigorous proliferative capabilities than NESC after being cultured for 24, 48, 72 and $96 \mathrm{~h}(P<0.05$; Fig. 3A). The relative proliferative ability of AC002454.1-RNAi and CDK6-RNAi were significantly lower than those in AC002454.1-NC, CDK6-NC and ESC $(P<0.05$; Fig. $3 \mathrm{~B}$ and $\mathrm{C})$. Relative proliferation in AC00254.1-CDNA and CDK6-CDNA were significantly higher than those in AC002454.1-NC, CDK6-NC and NESC $(P<0.05$; Fig. $3 \mathrm{D}$ and $\mathrm{E})$. There was no significant change between the negative lentiviral particles group and the blank control group $(P>0.05)$.

\section{Overexpression of AC002454.1 and CDK6 promote migration of endometrial stromal cells}

The cell scratch assay showed that the relative migration ability of AC002454.1-RNAi and CDK6-RNAi were significantly lower than those in AC002454.1-NC, CDK6-NC and ESC ( $P<0.01$; Fig. $4 \mathrm{~A}$ and C). The relative

Table 2 Immunohistochemical detection results of the CDK6 protein expression.

\begin{tabular}{lccr}
\hline Tissue samples & $\boldsymbol{n}$ & Positive $(\%)$ & Negative $(\%)$ \\
\hline Endometrium & & & \\
Ectopic & 30 & $20(66.7)^{* *}$ & $10(33.3)$ \\
Eutopic & 30 & $22(73.3)$ & $8(26.7)$ \\
Normal & 24 & $6(25.0)$ & $18(75.0)$ \\
\hline
\end{tabular}

The expression rate of CDK6 protein in the eutopic endometrium of EMS and normal endometrium.

${ }^{* *} P<0.001$ vs the normal endometrium. 

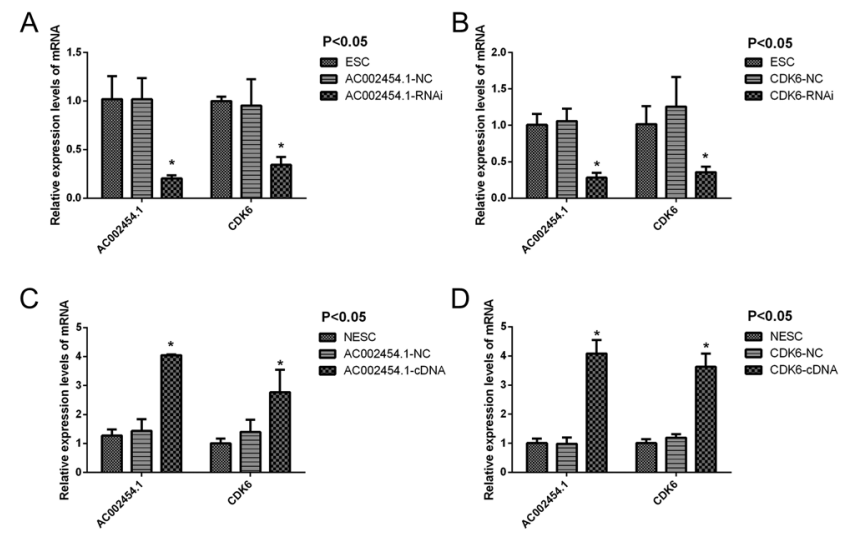

E

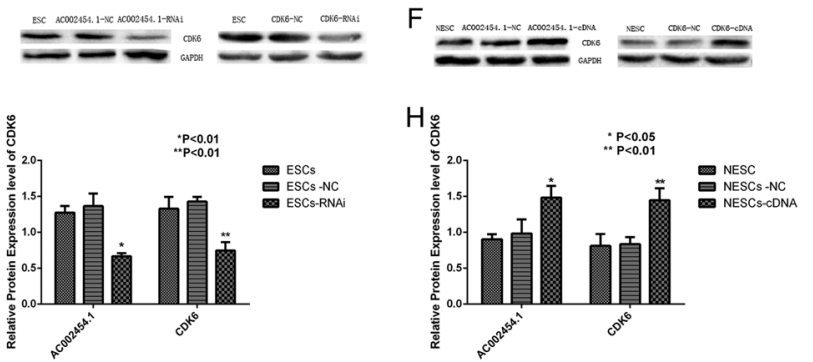

Figure 2 Expression of AC002454.1 and CDK6. (A, B, C and D) The relative expression levels of AC002454.1 and CDK6 after silencing AC002454.1 and CDK6 in eutopic endometrial cells from patients with EMS (ESC) and upregulating AC002454.1 and CDK6 in normal endometrial cells from a control group (NESC), respectively.

$* P<0.05$. (E and G) Western blotting of CDK6 proteins after silencing AC002454.1 and CDK6 in ESC. ${ }^{*} P<0.01,{ }^{* *} P<0.01$. ( $\mathrm{F}$ and $\mathrm{H}$ ) Western blotting after upregulating AC002454.1 and CDK6 in NESC. ${ }^{*} P<0.05,{ }^{* *} P<0.01$.

migration ability of AC00254.1-cDNA and CDK6-cDNA were significantly higher than those in AC002454.1-NC, CDK6-NC and NESC $(P<0.01$; Fig. 4B and D). There were no significant changes in the negative lentiviral particles group and the blank control group, respectively.

\section{Overexpression of AC002454.1 and CDK6 promote invasion of endometrial stromal cells}

Transwell invasion assays showed that the invasive ability of AC002454.1-RNAi and CDK6-RNAi were significantly lower than those of AC002454.1-NC, CDK6-NC and ESC $(P<0.01$; Fig. 5A and C). The invasive ability of AC00254.1-cDNA and CDK6-cDNA were significantly higher than those in AC002454.1-NC, CDK6-NC and NESC $(P<0.01$; Fig. 5B and D). There were no significant changes in the negative lentiviral particles group and the blank control group, respectively.

\section{Overexpression of AC002454.1 and CDK6 promote cell cycle transformation of endometrial stromal cells}

Flow cytometry showed that the proportion of cells in G0/G1 phase was increased significantly, while
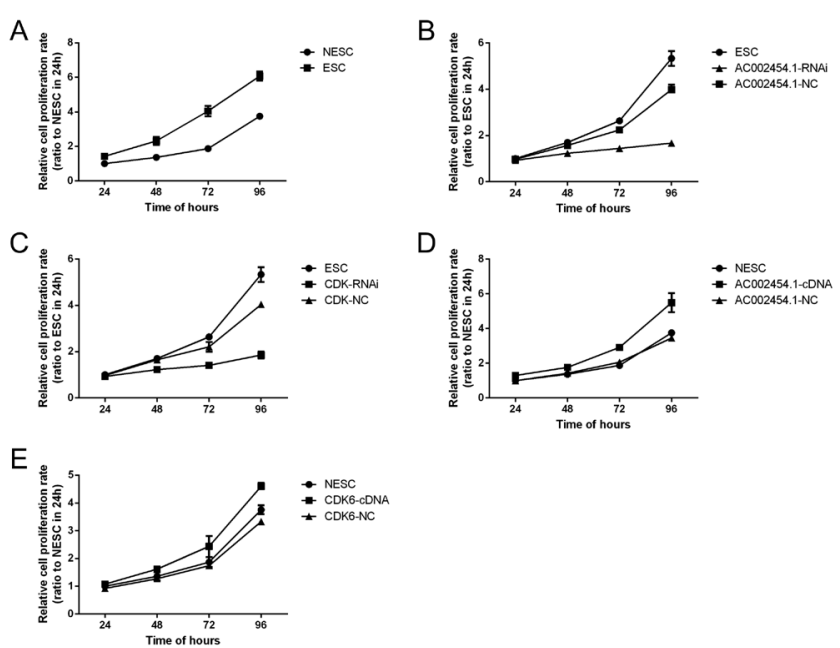

Figure 3 Cell proliferation results. (A) Cell proliferation ability of eutopic endometrial cells from patients with EMS (ESC) and normal endometrial cells from a control group (NESC) at basal level. (B, C, D and E) Cell proliferation ability after silencing AC002454.1 and CDK6 in ESC and upregulating AC002454.1 and CDK6 in NESC, respectively. ${ }^{*} P<0.05$.

the proportion of cells in $\mathrm{S}$ phase was decreased significantly in AC002454.1-RNAi and CDK6-RNAi when compared with AC002454.1-NC, CDK6-NC and ESC $(P<0.01$; Fig. 6A and C). In AC00254.1-CDNA and CDK6-CDNA, compared with AC002454.1-NC, CDK6-NC and NESC, the proportion of cells in G0/G1 phase was significantly decreased, while the proportion of $S$ phase was significantly increased $(P<0.01$; Fig. $6 \mathrm{~B}$ and $\mathrm{D})$. There were no significant changes in the negative lentiviral particles group and the blank control group, respectively.

\section{Discussion}

Previous research, using IncRNA microarrays, suggested that AC002454.1 was differentially expressed in EU and NE, while RT-PCR further suggested that the expression level of AC002454.1 was positively correlated with CDK6, the gene in a directly adjacent position (Wang et al. 2015). In the present study, we further confirmed that both the expression of AC002454.1 and CDK6 was significantly increased in EU and EC and that they showed positive correlation. Furthermore, after regulating the expression levels of AC002454.1 and CDK6, we identified numerous changes in biological behavior, including cell proliferation, migration and invasion. Both AC002454.1 and CDK6 can reduce the proportion of cells in G0/G1 phase and increase the proportion of cells in S phase. Consequently, our results indicate that both AC002454.1 and CDK6 can promote EMS by affect the biological behavior of endometrial stromal cells. The regulatory mechanism of AC002454.1 and CDK6 in EMS deserves further research attention. 


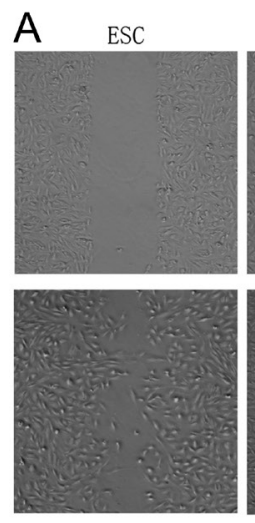

B
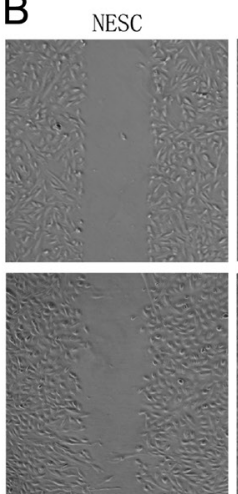
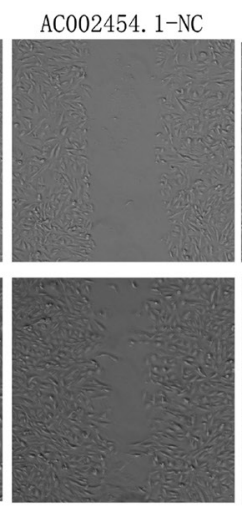

AC002454. 1-NC
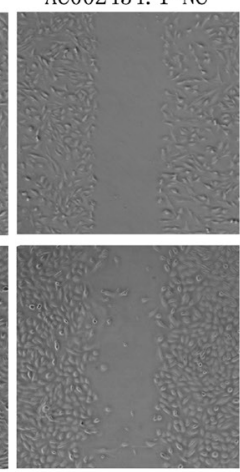
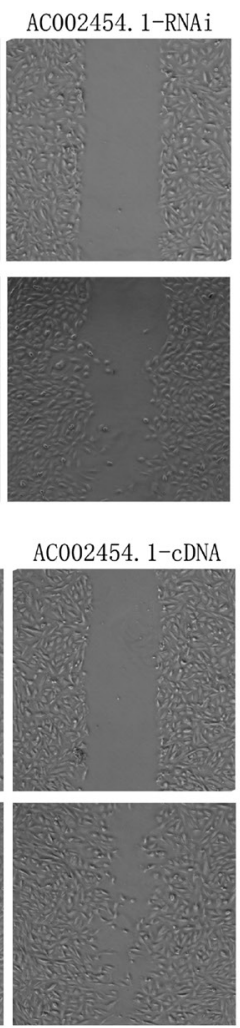
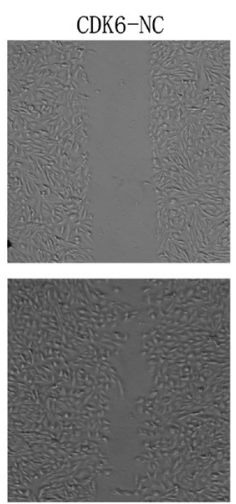

CDK6-NC

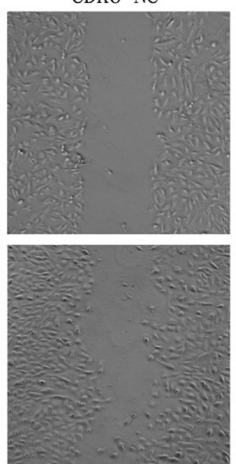

CDK6-RNAi

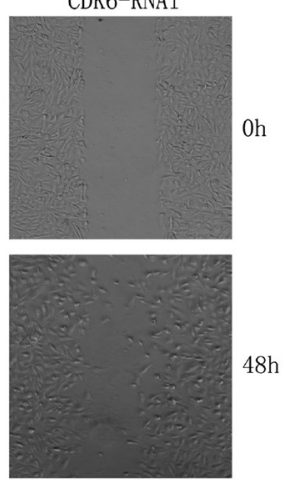

CDK6-CDNA

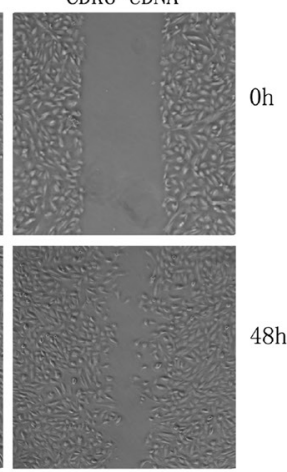

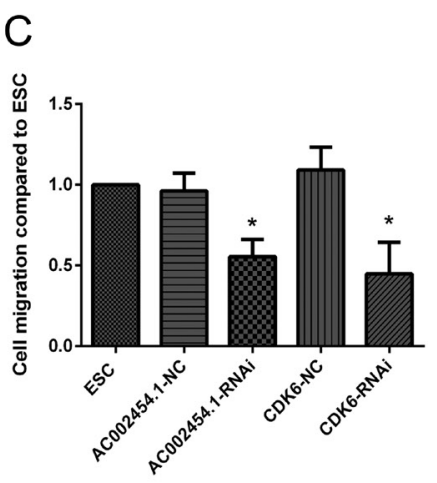

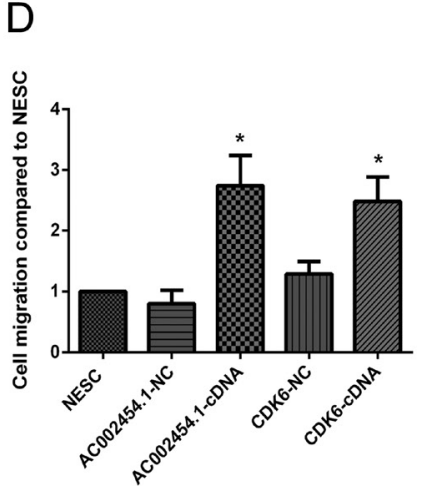

Figure 4 Results from the cell scratch assays. (A and C) Cell migration ability after silencing AC002454.1 and CDK6 in eutopic endometrial cells from patients with EMS (ESC). (B and D) Cell migration ability after upregulating AC002454.1 and CDK6 in normal endometrial cells from a control group (NESC). $* P<0.01$.

LncRNAs play a key role in the regulation of target genes in many diseases (Wu et al. 2018, Cremer et al. 2018). Furthermore, antisense IncRNAs can regulate target gene expression via a range of different mechanisms (Villegas \& Zaphiropoulos 2015, He et al. 2019). In the present study, our group showed that AC002454.1 may be one of the molecular targets involved in the origin of eutopic endometrial change in EMS. AC002454.1 is a natural antisense IncRNA with three transcripts: ENST00000424523; ENST00000452050 and ENST00000435695. It has been reported in the literature that ENST00000435695 is expressed at abnormally high levels in children with acute leukemia (AL) and that the expression level is related to the immunophenotype of children with AL. Downregulation of AC002454.1 expression can inhibit the proliferation of leukemia cells, affect the cell cycle, increase apoptosis rate and affect the expression of CDK6 (Cao et al. 2016). Other work has shown that ENST00000424523 is expressed at low levels in gastric cancer cells and inhibits both cell proliferation and migration (Luo et al. 2018). However, the role of ENST00000452050 has not been reported previously in the literature.

The present study found that ENST00000452050 is highly expressed in the eutopic endometrial cells of EMS. After upregulating the expression levels of AC002454.1, we identified that the cell proliferation, migration and invasion ability was enhanced and that the proportion of cells in G0/G1 phase was reduced, while the proportion in $\mathrm{S}$ phase was increased. After downregulating the expression levels of AC002454.1, we found that the cell proliferation, migration and invasion ability weakened, the proportion of cells in G0/G1 phase increased and that the proportion in S phase was reduced. Therefore, AC002454.1 plays an important role in the process of EMS by affecting the basic biological behavior of eutopic endometrium and that the molecular mechanism may be related to the regulation of CDK6 on the cell cycle. This provides new clues for the further exploration of mechanisms underlying EMS.

Previous pathway analysis, using chip technology, showed that the cell cycle pathway was ranked first and that CDK6 was the transcript showing the highest difference in this pathway (Wang et al. 2015). In addition, the role of cell cycle disorder in the occurrence and development of EMS is becoming a new focus for studying the mechanisms of EMS (Kobayashi et al. 2014). Incomplete and delayed transformation from the proliferative phase to the secretory phase is characteristic of the eutopic endometrium of EMS (Burney et al. 2009). This is accompanied by a persistent proliferative state of eutopic endometrial cells in the secretory phase. 
A
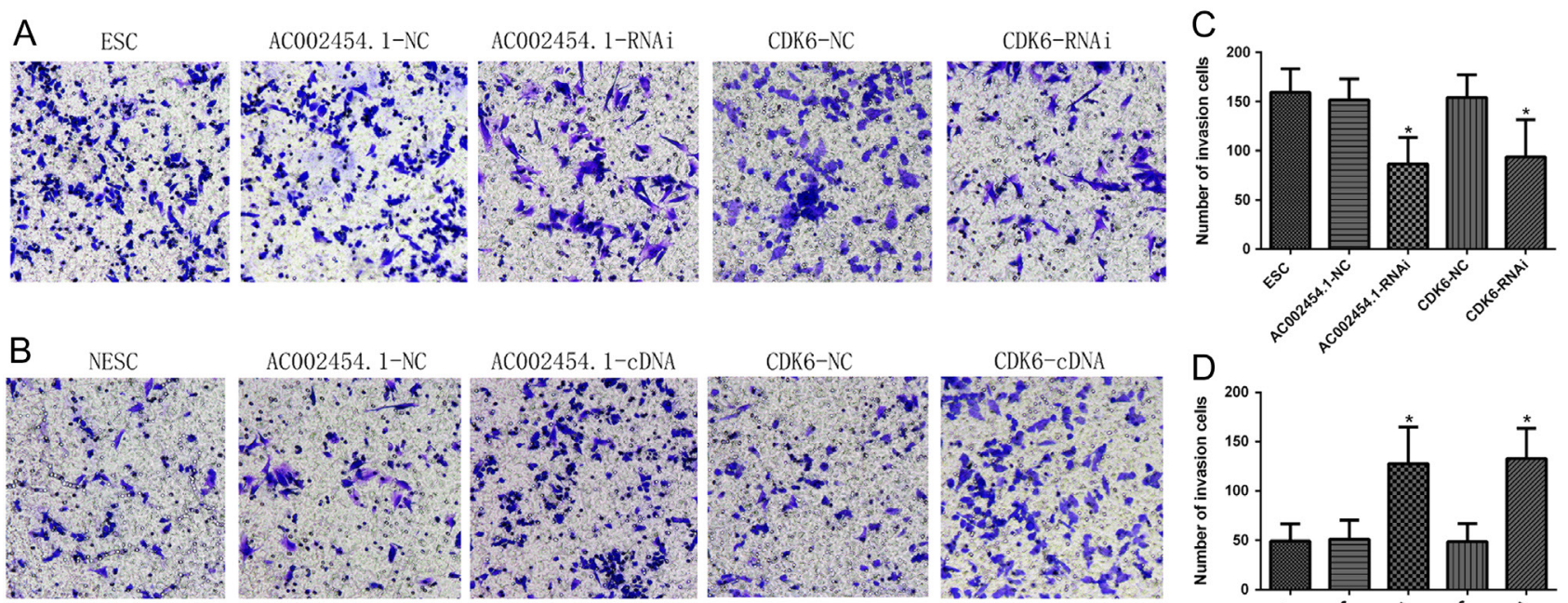

CDK6- $\mathrm{cDNA}$

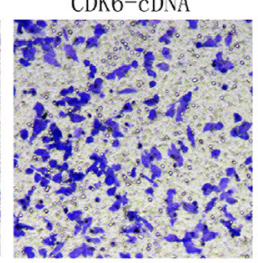

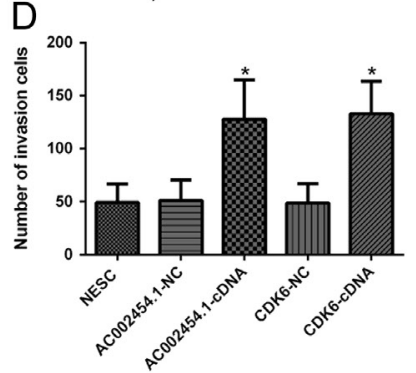

Figure 5 Transwell invasion assay results. (A and C) Cell invasive ability after silencing AC002454.1 and CDK6 in eutopic endometrial cells from patients with EMS (ESC). (B and D) Cell invasive ability after upregulating AC002454.1 and CDK6 in normal endometrial cells from a control group (NESC). ${ }^{*} P<0.01$.

A highly proliferative endometrium from the late secretory phase refluxing through the menstrual blood is more likely to form EMS. It has been reported that one option to search for new treatments for EMS is to search for genes associated with the cell cycle in order to inhibit the proliferation of endometrial cells. For example, anti-Mullerian hormone (AMH) can prolong the G1/G2 phase and shorten the S phase (Signorile et al. 2014). The G protein-coupled estrogen receptor agonist G-1 is known to arrest the cell cycle and accumulate in the G1 phase, inhibit the proliferation of endometrial cells, induce apoptosis and is expected to be able to alleviate and treat EMS in the future (Mori et al. 2015).
Recent studies have found that in addition to promoting cell proliferation, CDK6 also enhances the expression of VEGF-A, thereby inducing angiogenesis and endothelial cell growth, enhancing vascular permeability, although this action is not related to its kinase activity (Tigan et al. 2016). These biological characteristics are closely related to the process of EMS formation (Young et al. 2015).

The present study found that CDK6 is highly expressed in the eutopic endometrial cells of EMS. Upregulating the expression levels of CDK6 enhanced cell proliferation, migration and invasion ability, reduced the proportion of cells in G0/G1 phase and increased the proportion in $\mathrm{S}$ phase. Therefore, CDK6 can promote the progression
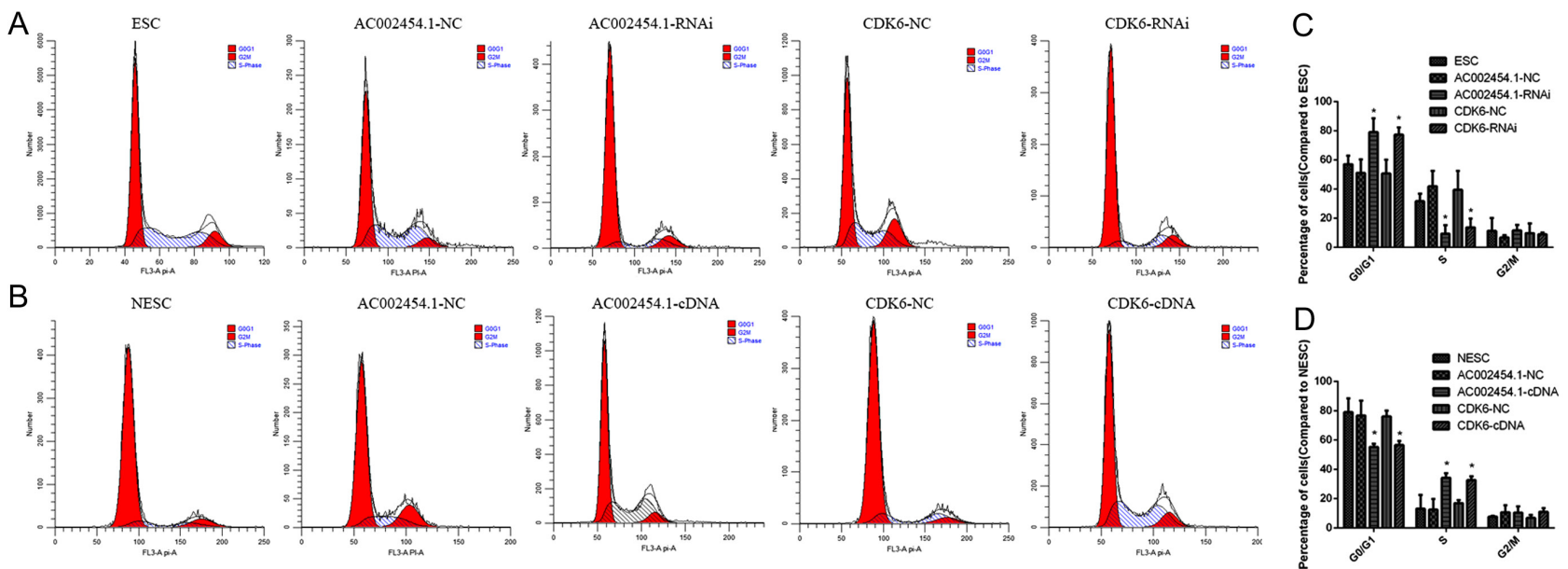

Figure 6 Flow cytometry results. (A and C). Cell cycle changes and the proportion of cells in G0/G1 phase and S phase after silencing AC002454.1 and CDK6 in eutopic endometrial cells from patients with EMS (ESC). (B and D) Cell cycle changes and the proportion of cells in G0/G1 phase and S phase after upregulating AC002454.1 and CDK6 in normal endometrial cells from a control group (NESC). ${ }^{*} P<0.01$. 
of EMS and indicates that CDK6 inhibitors may become a new method for treating EMS.

The present study suggested that the expression level of AC002454.1 is positively correlated with CDK6; we also observed that these genes are adjacent to each other in the genome, with an overlapping region. IncRNA acts in either a cis (neighboring gene interactions) or trans (interacting with remote transcriptional promoters or inhibitors) fashion to regulate gene expression at the transcriptional level (Bassett et al. 2014, Joung et al. 2017), in either a positive or negative manner. Therefore, it is reasonable to speculate that AC002454.1 and CDK6 may complement each other to form a IncRNA-mRNA duplex and participate in maintaining the stability of CDK6-mRNA and thus increase the protein expression level, thus causing cell cycle disorder in endometrial cells, enhancing their proliferative capacity and angiogenic ability. AC002454.1 may also affect the expression of CDK6, leading to cell cycle disorder and participate together in the pathogenesis of EMS. We have confirmed that both of them play a role in the progression of endometriosis, but whether there is a direct binding relationship between AC002454.1 and CDK6 or CDK6 regulates the expression of $\mathrm{AC} 002454.1$ through other feedback mechanisms still needs to explore in the future.

\section{Declaration of interest}

The authors declare that there is no conflict of interest that could be perceived as prejudicing the impartiality of the research reported.

\section{Funding}

This work was supported by the National Natural Science Foundation of China (grant number: 81771556).

\section{Author contribution statement}

J L, Y W and D B W conceived and designed this project and wrote the manuscript. J L, P C and $\mathrm{Y} M$ performed the experiments. S W, Y T and A N W participated in guiding the design of this research and supervised the whole experimental work. All authors read and approved the final manuscript.

\section{Acknowledgements}

The authors are grateful to everyone who provided help and support in the Department of Gynecology and the Central Laboratory of the Liaoning Cancer Hospital.

\section{References}

Bassett AR, Akhtar A, Barlow DP, Bird AP, Brockdorff N, Duboule D, Ephrussi A, Ferguson-Smith AC, Gingeras TR, Haerty W et al. 2014 Considerations when investigating IncRNA function in vivo. ELife 3 e03058. (https://doi.org/10.7554/eLife.03058)
Burney RO \& Giudice LC 2012 Pathogenesis and pathophysiology of endometriosis. Fertility \& Sterility 98 511-519. (https://doi.org/10.1016/j. fertnstert.2012.06.029)

Burney RO, Hamilton AE, Aghajanova L, Vo KC, Nezhat CN, Lessey BA \& Giudice LC 2009 MicroRNA expression profiling of eutopic secretory endometrium in women with versus without endometriosis. Molecular Human Reproduction 15 625-631. (https://doi.org/10.1093/molehr/ gap068)

Cao L, Xiao PF, Tao YF, Hu SY, Lu J, Zhao WL, Li ZH, Wang NN, Wang J, Feng $\mathbf{X}$ et al. 2016 Microarray profiling of bone marrow long noncoding RNA expression in Chinese pediatric acute myeloid leukemia patients. Oncology Reports 35 757-770. (https://doi.org/10.3892/ or.2015.4415)

Cremer S, Michalik KM, Fischer A, Pfisterer L, Jae N, Winter C, Boon RA, Muhly-Reinholz M, John D, Uchida S et al. 2018 Hematopoietic deficiency of the long non-coding RNA MALAT1 promotes atherosclerosis and plaque inflammation. Circulation Epub. (https://doi.org/10.1161/ CIRCULATIONAHA.117.029015)

Giudice LC \& Kao LC 2004 Endometriosis. Lancet 364 1789-1799. (https:// doi.org/10.1016/S0140-6736(04)17403-5)

He ZY, Wei TH, Zhang PH, Zhou J \& Huang XY 2019 Long noncoding RNA-antisense noncoding RNA in the INK4 locus accelerates wound healing in diabetes by promoting lymphangiogenesis via regulating miR181a/Prox1 axis. Journal of Cellular Physiology 234 4627-4640. (https:// doi.org/10.1002/jcp.27260)

Joung J, Engreitz JM, Konermann S, Abudayyeh OO, Verdine VK, Aguet F, Gootenberg JS, Sanjana NE, Wright JB, Fulco CP et al. 2017 Genome-scale activation screen identifies a IncRNA locus regulating a gene neighbourhood. Nature 548 343-346. (https://doi.org/10.1038/ nature23451)

Khorkova O, Hsiao J \& Wahlestedt C 2015 Basic biology and therapeutic implications of IncRNA. Advanced Drug Delivery Reviews 87 15-24. (https://doi.org/10.1016/j.addr.2015.05.012)

Kobayashi H, Uekuri C \& Shigetomi H 2014 Towards an understanding of the molecular mechanism of endometriosis: unbalancing epithelialstromal genetic conflict. Gynecological Endocrinology 30 7-15. (https:// doi.org/10.3109/09513590.2013.831832)

Lessey BA \& Kim JJ 2017 Endometrial receptivity in the eutopic endometrium of women with endometriosis: it is affected, and let me show you why. Fertility \& Sterility 108 19-27. (https://doi.org/10.1016/j. fertnstert.2017.05.031)

Ling Z, Wang X, Tao T, Zhang L, Guan H, You Z, Lu K, Zhang G, Chen S, Wu J et al. 2017 Involvement of aberrantly activated HOTAIR/EZH2/ miR-193a feedback loop in progression of prostate cancer. Journal of Experimental \& Clinical Cancer Research 36 159. (https://doi. org/10.1186/s13046-017-0629-7)

Loewen G, Jayawickramarajah J, Zhuo Y \& Shan B 2014 Functions of IncRNA HOTAIR in lung cancer. Journal of Hematology \& Oncology 7 90. (https://doi.org/10.1186/s13045-014-0090-4)

Luo Y, Liang M, Yao W, Liu J, Niu Q, Chen J, Liu Z, Li M, Shi B, Pan J et al. 2018 Functional role of IncRNA LOC101927497 in N-methyl-N'-nitro$\mathrm{N}$-nitrosoguanidine-induced malignantly transformed human gastric epithelial cells. Life Sciences 193 93-103. (https://doi.org/10.1016/j. Ifs.2017.12.007)

Lv X, Wang D, Ma Y \& Long Z 2018 Analysis of the oncogene BRAF mutation and the correlation of the expression of wild-type BRAF and CREB1 in endometriosis. International Journal of Molecular Medicine 41 1349-1356. (https://doi.org/10.3892/ijmm.2017.3342)

Martens-Uzunova ES, Bottcher R, Croce CM, Jenster G, Visakorpi T \& Calin GA 2014 Long noncoding RNA in prostate, bladder, and kidney cancer. European Urology 65 1140-1151. (https://doi.org/10.1016/j. eururo.2013.12.003)

Mckinnon B, Mueller M \& Montgomery G 2018 Progesterone resistance in endometriosis: an acquired property? Trends in Endocrinology \& Metabolism 29 535-548. (https://doi.org/10.1016/j.tem.2018.05.006)

Mori T, Ito F, Matsushima H, Takaoka O, Tanaka Y, Koshiba A, Kusuki I \& Kitawaki J 2015 G Protein-coupled estrogen receptor 1 agonist G-1 induces cell cycle arrest in the mitotic phase, leading to apoptosis in endometriosis. Fertility \& Sterility 103 1228-1235.e1. (https://doi. org/10.1016/j.fertnstert.2015.01.026)

Otto T \& Sicinski P 2013 The kinase-independent, second life of CDK6 in transcription. Cancer Cell 24 141-143. (https://doi.org/10.1016/j. ccr.2013.07.019) 
Paternot S, Colleoni B, Bisteau X \& Roger PP 2014 The CDK4/CDK6 inhibitor PD0332991 paradoxically stabilizes activated cyclin D3CDK4/6 complexes. Cell Cycle 13 2879-2888. (https://doi.org/10.4161/ 15384101.2014.946841)

Signorile PG, Petraglia F \& Baldi A 2014 Anti-Mullerian hormone is expressed by endometriosis tissues and induces cell cycle arrest and apoptosis in endometriosis cells. Journal of Experimental \& Clinical Cancer Research 33 46. (https://doi.org/10.1186/1756-9966-33-46)

Sundqvist J, Andersson KL, Scarselli G, Gemzell-Danielsson K \& Lalitkumar PG 2012 Expression of adhesion, attachment and invasion markers in eutopic and ectopic endometrium: a link to the aetiology of endometriosis. Human Reproduction 27 2737-2746. (https://doi. org/10.1093/humrep/des220)

Tigan AS, Bellutti F, Kollmann K, Tebb G \& SexI V 2016 CDK6-a review of the past and a glimpse into the future: from cell-cycle control to transcriptional regulation. Oncogene 35 3083-3091. (https://doi. org/10.1038/onc.2015.407)

Villegas VE \& Zaphiropoulos PG 2015 Neighboring gene regulation by antisense long non-coding RNAs. International Journal of Molecular Sciences 16 3251-3266. (https://doi.org/10.3390/ijms16023251)

Wang Y, Li Y, Yang Z, Liu K \& Wang D 2015 Genome-wide microarray analysis of long non-coding RNAs in eutopic secretory endometrium with endometriosis. Cellular Physiology and Biochemistry 37 2231-2245. (https://doi.org/10.1159/000438579)

Wu J, Zhu P, Lu T, Du Y, Wang Y, He L, Ye B, Liu B, Yang L, Wang J et al. 2018 The long noncoding RNA LncHDAC2 drives the self-renewal of liver cancer stem cells via activation of Hedgehog Signaling. Journal of Hepatology.

Xu YL, Wang DB, Liu QF, Chen YH \& Yang Z 2010 Silencing of cofilin-1 gene attenuates biological behaviours of stromal cells derived from eutopic endometria of women with endometriosis. Human Reproduction 25 2480-2488. (https://doi.org/10.1093/humrep/deq197)

Yan J, Dang Y, Liu S, Zhang Y \& Zhang G 2016 LncRNA HOTAIR promotes cisplatin resistance in gastric cancer by targeting miR-126 to activate the PI3K/AKT/MRP1 genes. Tumour Biology. (https://doi.org/10.1007/ s13277-016-5448-5)

Young VJ, Ahmad SF, Brown JK, Duncan WC \& Horne AW 2015 Peritoneal VEGF-A expression is regulated by TGF-beta1 through an ID1 pathway in women with endometriosis. Scientific Reports 5 16859. (https://doi. org/10.1038/srep16859)

Zhang Z, Chen P, Guo C, Meng X \& Wang D 2015 Effect of LIM kinase 1 overexpression on behaviour of endometriosis-derived stromal cells. Cell \& Tissue Research 359 885-893. (https://doi.org/10.1007/s00441014-2068-5)

Received 8 January 2019

First decision 13 February 2019

Revised manuscript received 26 February 2019

Accepted 18 March 2019 